Vega, A.J., Robles P., Y.A., Alvarado, O., \& Cedeño Mitre, C. (2021). Estructura de tallas, distribución y abundancia de Anadara tuberculosa (Bivalvia: Arcidae) en dos sistemas de manglar del Pacífico de Panamá. Revista de Biología Tropical, 69(2), 422-433. DOI 10.15517/rbt.v69i2.43934

DOI 10.15517/rbt.v69i2.43934

\title{
Estructura de tallas, distribución y abundancia de Anadara tuberculosa (Bivalvia: Arcidae) en dos sistemas de manglar del Pacífico de Panamá
}

\author{
Ángel Javier Vega ${ }^{1,2}$ \\ Yolani A. Robles P. ${ }^{1}$ \\ Onelys Alvarado ${ }^{1}$ \\ Carlos Cedeño Mitre ${ }^{3}$
}

1. Centro de Capacitación, Investigación y Monitoreo de la Biodiversidad en el Parque Nacional Coiba, Centro Regional Universitario de Veraguas, Universidad de Panamá (CCIMBIO-Coiba-UP), Santiago de Veraguas, República de Panamá; angel.vega@up.ac.pa, yolany.robles@up.ac.pa, onelys20panama@gmail.com

2. Estación Científica Coiba (Coiba-AIP), Clayton, Panamá, República de Panamá.

3. Programa de Maestría en Ecología y Manejo de Zonas Costeras, Centro Regional Universitario de Veraguas, Universidad de Panamá, Santiago de Veraguas, República de Panamá; cedenoc19@gmail.com

$$
\text { Recibido 22-IX-2020. Corregido 14-XII-2020. Aceptado 14-I-2021. }
$$

\section{ABSTRACT \\ Size structure, distribution, and abundance of Anadara tuberculosa (Bivalvia: Arcidae) in two mangrove systems from the Pacific Coast of Panama}

Introduction: Anadara tuberculosa is an economically important fishery resource of mangrove ecosystems in the tropical Eastern Pacific. Objective: The objective of this study was to analyze the size structure, distribution, and density of A. tuberculosa, in the Gulf of Montijo (GM) and Mangroves of David (MD) in the Panamanian Pacific, to assess the state of the resource in Panama's two Pacific mangrove systems. Methods: We analyzed Gulf of Montijo historical clam size records and densities from 2004 and 2020 . Hundred and seven $30 \mathrm{~m}^{2}$ permanent plots (51 from GOM and 56 from MOD) were set and sampled in 2016 at four time intervals (dry season, transition into rainy, rainy season and transition into dry season) to obtain clams sizes, densities and distributions. The plots were sampled again in 2019. Physical variables such as temperature $\left({ }^{\circ} \mathrm{C}\right)$, salinity (PSU) and $\mathrm{pH}$ from the water retained in the mangrove channels were also recorded. Nine transects were installed and sampled in 2019. Three at the seaward edge of the mangrove fringe, three at the inshore edge, and three half-way between the first two. Three quadrats were sampled at the beginning, middle and end of each transect. Results: Gulf of Montijo A. tuberculosa were found to larger but at lower densities than those from Mangroves of David. At each site, size comparison by sampling period, were statistically significant only for GOM, where larger sizes were found in March and July and smaller sizes in October-December. There was no correlation between the distributions of $A$. tuberculosa and temperature, salinity and $\mathrm{pH}$. A sustained decrease in A. tuberculosa densities was found for GOM (1.82 ind $/ \mathrm{m}^{2}$ in 2004 to $0.6 \mathrm{ind} / \mathrm{m}^{2}$ in 2019). This contrast with the predominantly larger clam sizes above $50 \mathrm{~mm}$ in total length and an increase in mean size of collected individuals (52.62 $\mathrm{mm}$ in 2004 to $59.28 \mathrm{~mm}$ in 2020). Specimens of $A$. tuberculosa were found up to slightly more than $2 \mathrm{~km}$ inshore from the main channel and within slightly more than $500 \mathrm{~m}$ from a secondary channel. Clam densities decreased and sizes increased in a seaward-inshore direction. Conclusions: For A. tuberculosa, the combination of size classes larger than $50 \mathrm{~mm}$ and low densities of individuals is evidence of population deterioration associated to fishery and environmental stressors.

Key words: black clam; Golfo de Chiriquí; fishery; density; piangua. 
Anadara tuberculosa (Sowerby, 1833), conocida localmente como concha negra, es un molusco bivalvo de la familia Arcidae. Se distribuye desde Baja California, en México, hasta la Bahía de Tumbes en el norte de Perú (Keen, 1971; MacKenzie, 2001). Vive en ambientes que están influenciados por lo ciclos de mareas y donde la salinidad sea superior a 12 ups, especialmente asociada a las raíces de Rhizophora mangle, R. harrisonni y Pelliciera rizophorae (Campos, Fourtnier, \& Soto, 1990; Cruz \& Jiménez, 1994; Vega, 1994; Silva \& Bonilla, 2001).

La concha negra, se considera uno de los principales recursos pesqueros bentónicos asociados a los sistemas de manglar en toda su área de distribución (MacKenzie, 2001). Su importancia económica se pone de manifiesto en relación con los niveles de explotación a los que ha estado sometida. En Colombia se estimó una disponibilidad del recurso de 1500.00 toneladas para un área de $30 \mathrm{~km}^{2}$ (Squires, Esteves, Barona, \& Mora, 1975), una captura máxima sostenible de $4897960 \mathrm{~kg}$ (Cruz \& Borda, 2003) y que la pesquería de la piangua, nombre local con el que se le conoce, es no planificada y sin controles, con un $55 \%$ de las capturas por debajo de la talla mínima legal, donde la caída de las capturas se asocia a la sobrepesca combinada con los efectos del niño (Cruz \& Borda, 2003; Borda \& Cruz, 2004). En este mismo sentido, para Costa Rica, se ha reportado que el pequeño tamaño de las poblaciones, el fácil acceso al recurso y su valor económico inciden en los procesos de sobreexplotación (Stern-Pirlot \& Wolff, 2006) y para Perú, que la extracción de individuos en tallas que no han alcanzado la madurez sexual puede estar reduciendo las capturas y las densidades de la concha negra (PantaVélez, Bermúdez-Medranda, Mero, Arrieche, \& Acosta-Balbás, 2020).

Los sistemas de explotación no regulados o con regulaciones débiles afectan la abundancia del recurso, las tallas de extracción y comercialización, y conducen a la sobrepesca y sobreexplotación. En este sentido indicadores poblacionales como estructura de tallas y densidad reflejan caídas en sus valores en El Salvador (Flores, Romero, \& Córdova, 2011), Costa Rica (Silva \& Bonilla, 2001; Stern-Pirlot \& Wolf, 2006; Silva-Benavides \& Bonilla, 2015), Colombia (Lucero, Cantera, \& Neira, 2012), Ecuador (Panta-Vélez et al., 2020) y Perú (Ordinola, Alemán, \& Montero, 2020),

Los patrones de distribución y abundancia de la concha negra se han asociado a factores como composición y estructura del sustrato, su dureza, factores oceanográficos y pesqueros (Vega, 1994; Silva \& Bonilla, 2001; Guilbert, 2007; Silva-Benavides \& Bonilla, 2015). Algunos estudios indican que la salinidad influye en la distribución y abundancia de la concha negra, encontrándose en salinidades por encima de 10-12 ups (Vega 1994; Silva \& Bonilla, 2001), ya que salinidades bajas favorecen su mortalidad (Mendoza et al., 2017), mientras que altas benefician la reproducción y el crecimiento (Silva \& Bonilla, 2001; Panta-Vélez et al., 2020). Otros autores señalan que la concha negra se distribuye hasta los $200 \mathrm{~m}$ desde el borde del canal hacia la parte interna del manglar (Campos et al., 1990; MacKenzie, 2001) y que la densidad aumenta hacia los estratos interiores (Mora-Sánchez, 2012); que permanece igual (Ordinola et al., 2020) o que disminuye (Guilbert, 2007; Flores et al., 2011).

El objetivo del estudio fue analizar la estructura de tallas, distribución y densidad de Anadara tuberculosa, en el Golfo de Montijo y Manglares de David, para evaluar el estado del recurso en los dos sistemas de manglar del Pacífico de Panamá.

\section{MATERIALES Y METODOS}

El estudio se desarrolló en dos de los principales sistemas de manglar del Pacífico panameño: El Golfo de Montijo, en Veraguas y los mangares de David en Chiriquí (Fig. 1).

Golfo de Montijo (GM): Es un área protegida y sitio Ramsar, definido por un polígono cuyas coordenadas son $\left(7^{\circ} 56^{\prime} 4^{\prime \prime}-7^{\circ} 59^{\prime} 30^{\prime \prime} \mathrm{N} \&\right.$ $\left.81^{\circ} 17^{\prime} 33^{\prime \prime}-81^{\circ} 1^{\prime} 40^{\prime \prime} \mathrm{W}\right)$; (7³8'20"-7०36'29" $\left.\mathrm{N} \& 80^{\circ} 58^{\prime} 40^{\prime \prime}-81^{\circ} 133^{\prime} 37^{\prime \prime} \mathrm{W}\right)$ (Resolución 


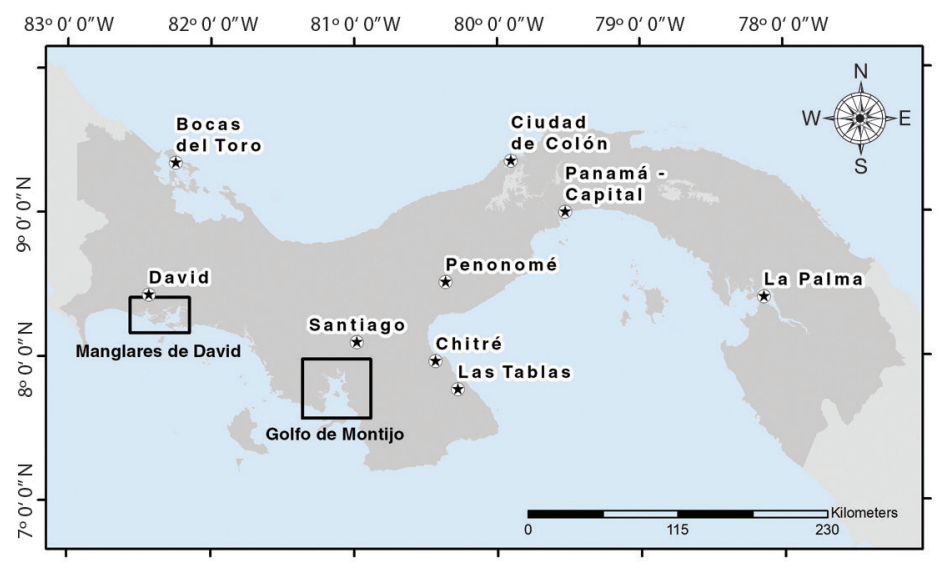

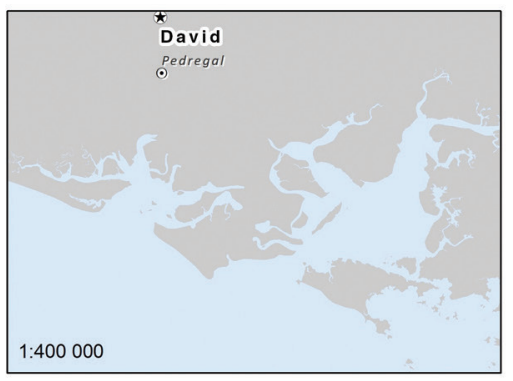

Manglares de David

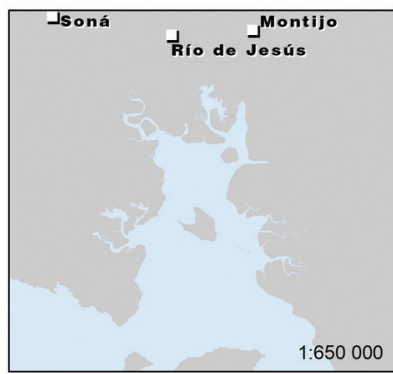

Golfo de Montijo

Fig. 1. Zona de estudio, Golfo de Montijo y Manglares de David, Panamá.

Fig. 1. Study zone, Gulf of Montijo and Mangroves of David, Panama.

DAPVS 0001 2016, del 25 de enero de 2016 publicada en la Gaceta Oficial Digital $\mathrm{N}^{\circ}$ 27972, del 9 de febrero de 2016) y es un Humedal de Importancia Internacional (Sitio Ramsar). Presenta un rico ambiente acuático de esteros y deltas, playas, zonas lodosas, arenosas y manglares (CATHALAC, 2007). Los manglares y los bosques de Ciénega constituyen $313 \mathrm{~km}^{2}$, de los cuales $279 \mathrm{~km}^{2}$ pertenecen a los manglares ubicados frente a una lámina de agua que tiene una superficie de $534.9 \mathrm{~km}^{2}$ (Cámara et al., 2004).

Es una importante zona de pesca del Pacífico panameño con una pesquería multiespecífica: concha negra (Jordán \& Gómez, 2006); langosta (Vega, Robles, \& Gil, 2013); corvinas, pargos y sierra (Vega, Robles, Boniche, \& Rodríguez, 2008; Bonilla, Robles, \& Vega, 2013; Vega, Quezada, \& Robles, 2013; Vega, Robles, \& Godoy, 2015), poliquetos (Vega,
Robles, \& Torres, 2014) y cangrejos (Vega, Mena. \& Robles, 2018).

Manglares de David (MD): Fue declarada área protegida a través de Decreto Municipal 21 del 6 de junio de 2007, por medio del cual se adoptaron disposiciones para la protección del ambiente y los manglares en las costas del distrito de David. Las coordenadas son $\left(8^{\circ} 22^{\prime} 47^{\prime \prime} \mathrm{N}-82^{\circ} 26^{\prime} 50^{\prime \prime} \mathrm{W}\right.$; 8 $8^{\circ} 20^{\prime} 09^{\prime \prime}$ $\mathrm{N}-82^{\circ} 12^{\prime} 56^{\prime \prime} \mathrm{W} ; 8^{\circ} 13^{\prime} 49^{\prime \prime} \mathrm{N}-82^{\circ} 19^{\prime} 12^{\prime \prime} \mathrm{W}$ $\left.\& 8^{\circ} 17^{\prime} 19^{\prime \prime} \mathrm{N}-82^{\circ} 28^{\prime} 49^{\prime \prime} \mathrm{W}\right)$. En esta zona desembocan varios ríos, como son: David, Chiriquí, Chico y Chorcha (Díaz del Olmo, Cámara-Artigas, \& Martínez-Batlle, 2004). Las principales actividades económicas que se desarrollan en este sistema son: la pesca artesanal, la recolección de conchas, cangrejos y la extracción de productos forestales (Tovar, 2008; Vega et al., 2015). 
Densidad y distribución: Los muestreos de densidades se realizaron a través de cuadrantes (Vega, 1994). Para el GM se analizaron datos históricos, no publicados, para densidades del 2004, 2013, 2016 y 2019 y para tallas de capturas comerciales del 2004, 2013, 2015, 2016, 2019 y 2020. En el 2016 se realizaron muestreos de forma simultánea en el GM y $\mathrm{MD}$, en periodo seco (marzo), transición (junio), lluvioso (octubre) y transición (diciembre). En cada zona se establecieron cuadrantes de $5 \mathrm{~m}$ x $6 \mathrm{~m}\left(30 \mathrm{~m}^{2}\right)$ bordeando la línea de manglar con el canal, donde regularmente se extrae el molusco, para el GM 51 y en MD 56. En cada sitio, donde se realizó el muestreo, se registró: coordenadas geográficas con el uso de un GPS, salinidad (ups), temperatura $\left({ }^{\circ} \mathrm{C}\right)$ y $\mathrm{pH}$, del agua del canal y retenida en el manglar, mediante una sonda multiparamétrica YSI 30. En el 2016 se establecieron tres cuadrantes fijos para monitoreo en el GM, a los cuales se les dio seguimiento por 11 meses (2016-2017), y fueron muestreados nuevamente en el 20192020, por 10 meses seguidos.

En el 2019, se establecieron seis transectos, en el GM, entre el límite del manglar con el canal principal y el límite con tierra firme, con tres estratos por transecto y tres cuadrantes de $30 \mathrm{~m}^{2}$ por estrato. Se contabilizó el número de conchas por cuadrante, la longitud total del molusco, medida desde la parte anterior del ejemplar con la parte ventral hacia arriba; las coordenadas geográficas y la distancia de cada estrato al canal principal y canal secundario más cercano.

Los datos fueron analizados con el programa IBM SPSS Statistics 25. Para probar la normalidad y Homocedasticidad de los datos se aplicaron las pruebas Kolmogorov-Smirnov y Levene, respectivamente. En ausencia de normalidad y homocedasticidad se aplicó estadística no paramétrica de la siguiente manera: para los muestreos del 2016 se comparó la densidad, temperatura, $\mathrm{pH}$, salinidad y tallas entre localidades (U de Mann-Whitney). Dentro de cada localidad se compararon las tallas entre periodos (meses) (Kruskal-Wallis y la prueba Post Hoc Bonferroni de Kruskal-Wallis), además se analizó la asociación entre densidad, temperatura, $\mathrm{pH}$ y salinidad a través de la correlación por rangos de Spearman. Se compararon las tallas y densidades entre años (2004 al 2020), así como las tallas y densidades entre estratos (muestreos del 2019) (Kruskal-Wallis y la prueba Post Hoc Bonferroni de Kruskal-Wallis). En todos los casos para $\alpha=0.05$ (Zar, 2009).

\section{RESULTADOS}

Parámetros ambientales: Presentaron un patrón asociado al comportamiento de las temporadas climáticas, con valores altos en la temporada seca y bajos en la lluviosa, excepto el $\mathrm{pH}$, en $\mathrm{MD}$, que presentó su valor más bajo en la temporada seca. El $\mathrm{pH}$, la temperatura y la salinidad resultaron significativamente diferentes al comparar GM con MD, tanto para valores registrados en el agua del canal como del agua retenida en el manglar. Los valores de $\mathrm{pH}$ resultaron superiores en MD, a diferencia de la temperatura y la salinidad que resultaron superiores en GM (U de Mann-Whitney, P < 0.05) (Fig. 2).

Tallas: Los ejemplares extraídos en el GM mostraron una mayor talla (Media = $56.37 \pm 10.19 \mathrm{~mm}, \mathrm{~N}=3$ 360) comparado con los extraídos en los MD (Media $=50.56$ $\pm 8.05, \mathrm{~N}=4443$ ) (U de Mann-Whitney, $\mathrm{P}<$ 0.05). La comparación de tallas por periodo de muestreo, dentro de cada localidad, resultó significativa solo para el GM (Kruskal Wallis $=28.88 ; \mathrm{P}<0.05)$, donde las mayores tallas se presentaron en marzo y junio y las menores en octubre-diciembre (Bonferroni de Kruskal Wallis, $\mathrm{P}<0.05$ ).

La comparación de tallas por años, para el GM, mostró tres grupos (Kruskal Wallis, $\mathrm{P}<0.05)$. Las menores se presentaron en el 2004 (Media $=52.62 \pm 8.00 \mathrm{~mm}, \mathrm{~N}=2639$ ), seguido del 2013 (Media $=58.39 \pm 8.47 \mathrm{~mm}$, $\mathrm{N}=2$ 037) y el último grupo, con las mayores tallas, en el 2015 (Media $=59.32 \pm 8.20 \mathrm{~mm}$, $\mathrm{N}=838), 2016($ Media $=59.79 \pm 9.56 \mathrm{~mm}, \mathrm{~N}$ $=10$ 878), $2019($ Media $=59.09 \pm 7.96 \mathrm{~mm}, \mathrm{~N}$ $=8$ 414) y $2020($ Media $=59.28 \pm 7.45 \mathrm{~mm}$, 


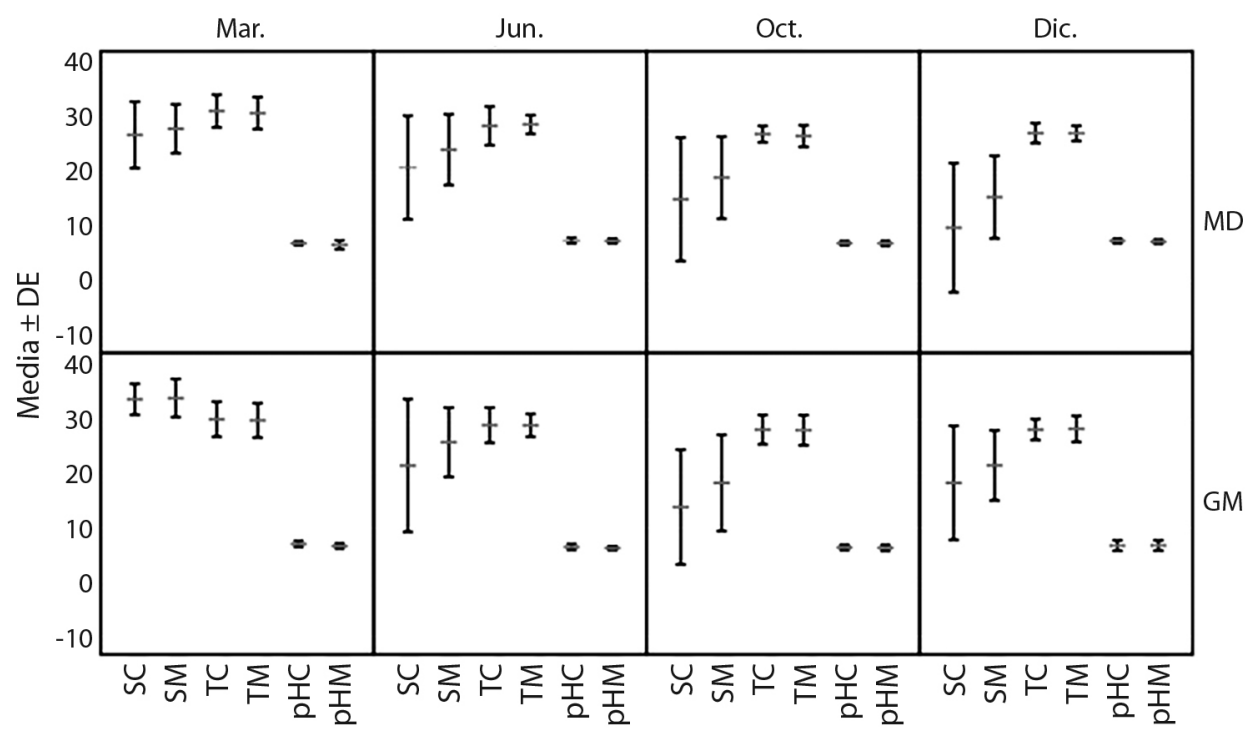

Fig. 2. Valores promedios mensuales de la temperatura, la salinidad y el pH, estimados para el Golfo de Montijo (GM) y Manglares de David (MD) a partir de los valores registrados en el manglar (M) y en el canal frente al manglar (C). Muestreos realizados en el 2016. Marzo: temporada seca, jun: transición, oct: lluviosa y dic. transición.

Fig. 2. Mean monthly average temperature, salinity, and pH for the Gulf of Montijo (GM) and Mangroves of David (MD), from values registered in the mangrove $(\mathrm{M})$ and the channel in front of the mangrove $(\mathrm{C})$. Sampling carried out during the year 2016: dry, June: transition, October: rainy and December: transition seasons.

$\mathrm{N}=2$ 609) (Bonferroni de Kruskal Wallis, $\mathrm{P}<$ 0.05) (Fig. 3).

Distribución: La concha negra en el GM se encontró en sitios con salinidades entre 9 y 39 ups, temperatura de 24.8 a $35.9{ }^{\circ} \mathrm{C} \mathrm{y} \mathrm{pH}$ de 6.19 a 8.09. Para MD con salinidades entre 5 y 33 ups, temperatura de 25.9 a $33.6{ }^{\circ} \mathrm{C} \mathrm{y} \mathrm{pH} \mathrm{de}$ 5.78 a 8.37 (Fig. 4). El análisis de correlación entre estos parámetros y las densidades resultó no significativo en las dos localidades (Spearman, $\mathrm{P}>0,05)$. Atendiendo a la distancia de distribución, se analizamos dos escenarios, el primero la densidad desde el borde del manglar en el canal principal hasta el límite del manglar con tierra firme, que representó distancias entre 144 y $2324 \mathrm{~m}$ (Media $=754.33 \mathrm{~m}$ ) y el segundo escenario, la distancia del estrato al borde de manglar del canal secundario más cercano, que varió entre 142 a $675 \mathrm{~m}($ Media $=396.17 \mathrm{~m})$.

La comparación de tallas y densidades por estratos fue significativa (Kruskal Wallis, $\mathrm{P}<$
0.05), con las mayores tallas en el estrato final comparado con los estratos intermedio e inicial, entre los cuales no se encontró diferencias significativas, inverso al comportamiento de las densidades que disminuyeron hacia el estrato final (Bonferroni de Kruskal-Wallis, $\mathrm{P}<0.05$ ).

Densidad: Para MD la densidad (Media $=0.66$ conchas $/ \mathrm{m}^{2} \pm 0.40, \mathrm{~N}=224$ ) resultó superior a la estimada para el GM (Media $=0.54$ conchas $\left./ \mathrm{m}^{2} \pm 0.50, \mathrm{~N}=204\right)(\mathrm{U} \mathrm{de}$ Mann-Whitney, $\mathrm{P}<0.05)$. Dentro de cada localidad, la densidad por mes de muestreo no reflejó diferencias significativas (KruskalWallis, $\mathrm{P}>0.05$ ).

Para el GM, la comparación de densidades por años mostró diferencias significativas (Kruskal-Wallis, $\mathrm{P}<0.05$ ). Los valores fueron 1.82 conchas $/ \mathrm{m}^{2}, 0.70$ conchas $/ \mathrm{m}^{2}, 0.54$ conchas $/ \mathrm{m}^{2}$ y 0.60 conchas $/ \mathrm{m}^{2}$, para 2004, 2013, 2016 y 2019, respectivamente, con diferencias significativas entre todos los años, excepto 


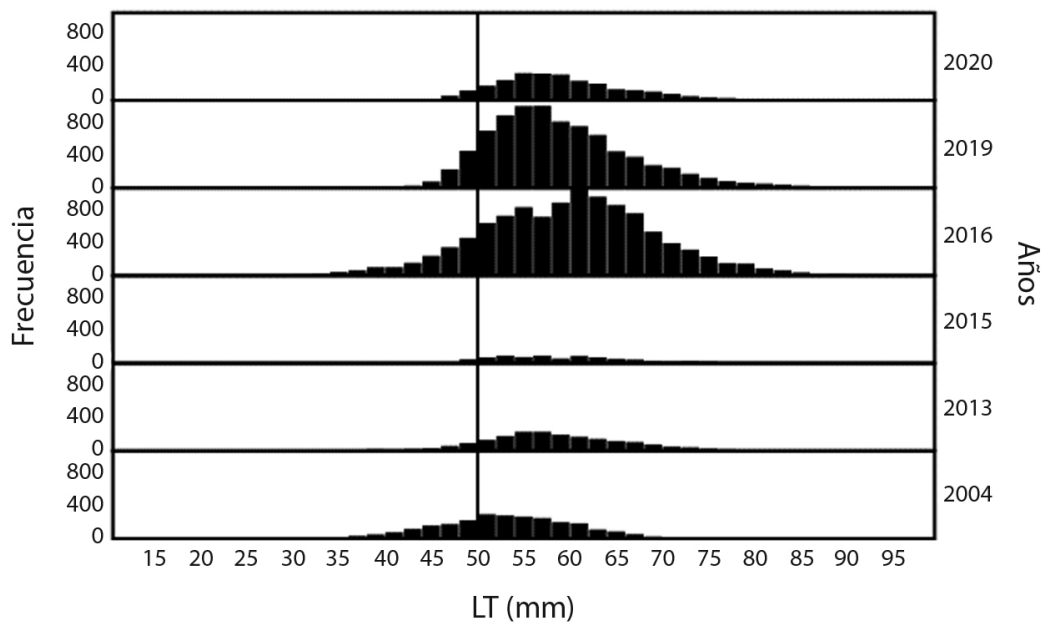

Fig. 3. Estructura de tallas de Anadara tuberculosa capturadas en el Golfo de Montijo y medidas en los puntos de desembarque o acopio. Muestreos realizados entre el 2004 y 2020. La línea negra indica la talla mínima legal de captura para el Golfo de Montijo, Panamá. LT: largo total.

Fig. 3. Size structure of Anadara tuberculosa captured in the Gulf of Montijo and landing sites between 2004 and 2020 . The black line indicates minimum legal size for the Gulf of Montijo, Panama. LT: total length.

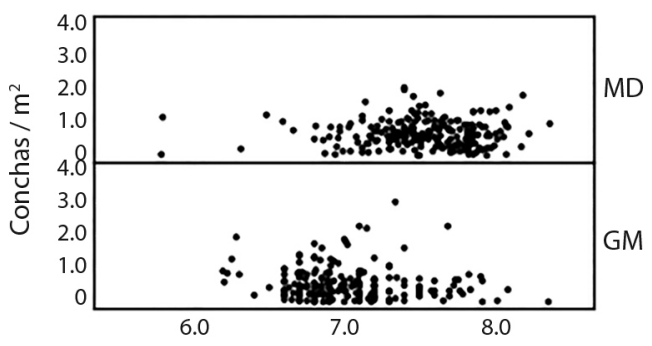

$\mathrm{pH}$
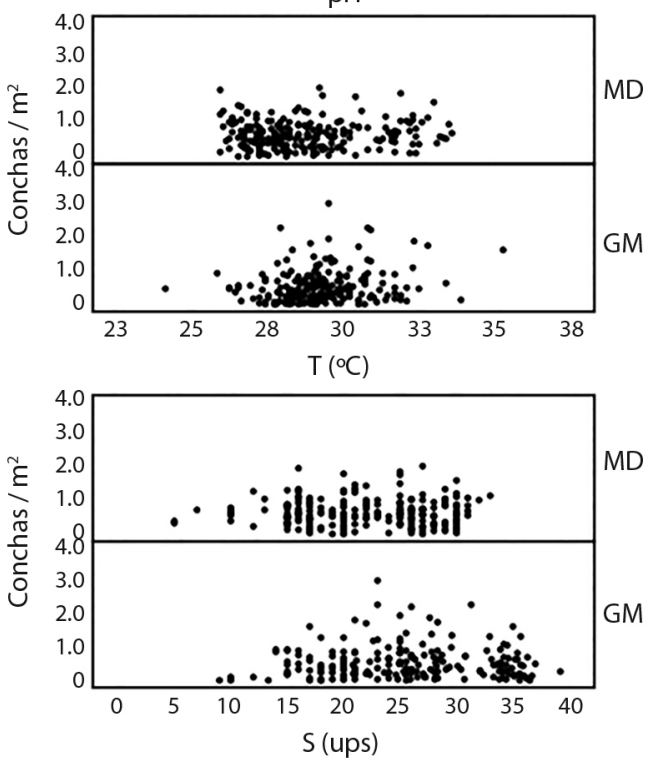

disminución sostenida a lo largo de los meses (Kruskal Wallis, $\mathrm{P}<0.05$ ); sin embargo, la comparación de densidades en los cuadrantes fijos entre los dos periodos de muestreo, para el GM, no mostró diferencias significativas (U de Mann-Whitney, P > 0.05) (Fig. 5).

En la Tabla 1 se presentan los resultados de densidades, longitud total y área de muestreo para Panamá comparada con otras localidades de la región. Las tallas aumentaron de 52.62 $\mathrm{mm}$ en el 2004 a $59.28 \mathrm{~mm}$ en el 2020, contrario al comportamiento de las densidades, que disminuyeron de 1.82 conchas $/ \mathrm{m}^{2}$ a 0.60 conchas $/ \mathrm{m}^{2}$ en el mismo periodo.

Fig. 4. Distribución y densidad (D) de Anadara tuberculosa en función de la temperatura, salinidad y pH. Muestreos realizados en el Golfo de Montijo y Manglares de David, Panamá, en el 2016. Los valores corresponden a muestras de agua tomadas en cada cuadrante dentro del manglar.

Fig. 4. Distribution and density (D) of Anadara Tuberculosa as a function of temperature, salinity and $\mathrm{pH}$. Sampling from Gulf of Montijo and Mangroves of David, Panama, in year 2016. Data correspond to water samples taken from each quadrant within the mangrove. 


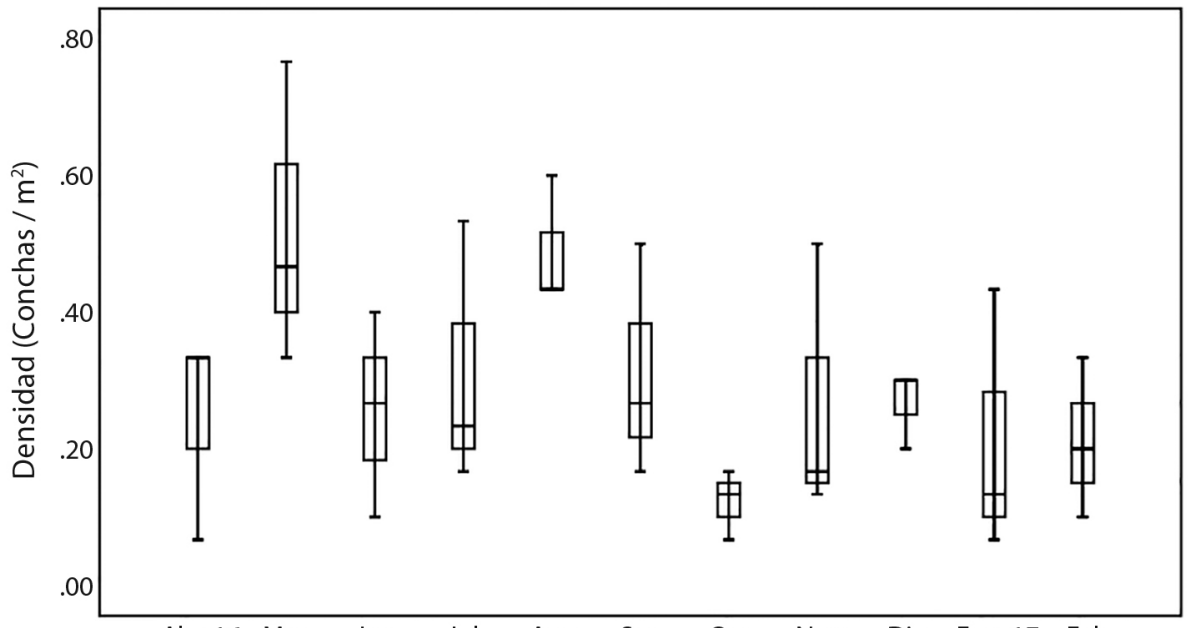

Abr. 16 May. Jun. Jul. Ago. Set. Oct. Nov. Dic. Ene. 17 Feb.

Mes

Fig. 5. Comportamiento de densidad en cuadrantes permanentes muestreados en el 2016-2017 y 2019-2020. Muestreos realizados en el Golfo de Montijo, Panamá.

Fig. 5. Density within permanent quadrants sampled in 2016-2017 and 2019-2020 in the Gulf of Montijo, Panama.

TABLA 1

Valores de densidad (D) en conchas $/ \mathrm{m}^{2}$, y tallas (LT) reportados para diferentes localidades del Pacífico Oriental Tropical

TABLE 1

Density (D) values as ind $/ \mathrm{m}^{2}$ and size (LT) reported from different sites in the Eastern pacific of Panama

\begin{tabular}{ccllc} 
D & LT $(\mathrm{mm})$ & \multicolumn{1}{c}{ Localidad } & \multicolumn{1}{c}{ Autor } & $\mathrm{A}\left(\mathrm{m}^{2}\right)$ \\
1.3 & 41.7 & Tumbes, Perú & Ordinola et al., 2019 & 1 \\
1 & 37.3 & Tumbes, Perú & Ordinola et al., 2020 & 1 \\
2.7 & 41.8 & El Morro, Ecuador & Gamboa-Landivar, 2019 & 1 \\
0.72 & 42.95 & Bazan y Nerete, Colombia & Espinosa et al., 2010 & 100 \\
0.64 & 52.21 & Bahia Málaga, Colombia & Lucero et al., 2012 & 100 \\
0.9 & 43.3 & Purruja, Golfo Dulce, Costa Rica & Silva \& Bonilla, 2001 & 100 \\
2.8 & 42.5 & Golfito, Costa Rica & Silva-Benavides \& Bonilla, 2015 & 100 \\
0.14 & 48.4 & Playa Blanca, Costa Rica & Silva-Benavides \& Bonilla, 2015 & 25 \\
1.1 & 53.1 & Térraba-Sierpe, Costa Rica & Campos et al., 1990 & 100 \\
1.72 & 47.9 & Térraba-Sierpe, Costa Rica & Vega, 1994 & 16 \\
0.12 & 46.5 & Bahía de Jiquilisco, El Salvador & Portillo et al., 2011 & 100 \\
$<0.4$ & $*$ & Las Perlas, Panamá & Gilbert, 2007 & 9 \\
1 & 48.08 & Boca de Trinidad, Montijo, Panamá & Jordán \& Gómez, 2006 & 30 \\
0.54 & 59.07 & Golfo de Montijo, Panamá & Este estudio (muestreo 2016) & 30 \\
0.39 & 59.79 & Golfo de Montijo, Panamá & Este estudio (muestreo 2019) & 30 \\
0.66 & 50.56 & Manglares de David, Panamá & Este estudio (muestreo 2016) & \\
\hline
\end{tabular}

A: área de muestreo. *. No se indicó las tallas. / A: sampling area. * sizes not available. 


\section{DISCUSIÓN}

Los MD y el GM, son dos sistemas estuarinos con una cobertura importante de manglar que albergan poblaciones de diferentes organismos sometidos a explotación pesquera (CATHALAC, 2007), entre ellos Anadara tuberculosa señalada en el 2006, como un recurso no sobrexplotado en función de la estructura de tallas estimada: promedio 48.1 $\mathrm{mm}$, con mayor frecuencia de individuos entre 40.0 y $60.0 \mathrm{~mm}$ y densidad estimada para la localidad de Morrito de 1.00 concha $/ \mathrm{m}^{2}$ (Jordán \& Gómez, 2006). Esta densidad contrasta con lo estimado en el 2004 para todo el Golfo de Montijo de 1.82 conchas $/ \mathrm{m}^{2}$, valor que fue decreciendo hasta el 2019, donde se estima una densidad de 0.60 conchas $/ \mathrm{m}^{2}$. En Manglares de David, a pesar de que no se tienen registros extensos, una estimación realizada por una empresa consultora (Planeta, 2012) comunicó 0.85 conchas $/ \mathrm{m}^{2}$, lo que resulta ligeramente superior a lo estimado para el 2016 para la misma localidad (0.66 conchas $\left./ \mathrm{m}^{2}\right)$. Para el archipiélago de las Perlas, en el Golfo de Panamá, se estimaron densidades inferiores a 0.4 conchas $/ \mathrm{m}^{2}$ (Guilbert, 2007), de los más bajos para el Pacífico panameño, lo que sugiere que las poblaciones de concha negra en el Pacífico panameño han disminuido en los últimos 15 años.

Para otras localidades del Pacífico Oriental Tropical se han estimado densidades variables. En Térraba-Sierpe se estimó una densidad promedio de 1.1 concha $/ \mathrm{m}^{2}$ (Campos et al., 1990) y cuatro años más tarde, en la misma localidad, 1.72 conchas $/ \mathrm{m}^{2}$ (Vega, 1994). En los manglares de Golfito, 2.99 conchas $/ \mathrm{m}^{2}$, ámbito de 0.95 a 6.0 conchas $/ \mathrm{m}^{2}$ y para el manglar de Playa Blanca una densidad promedio de 0.14 conchas $/ \mathrm{m}^{2}$, con valores entre 0.08 y 0.23 conchas/ $\mathrm{m}^{2}$ (Silva-Benavides \& Bonilla, 2015). Para El Salvador las densidades se estimaron entre 0.12 y 0.13 conchas $/ \mathrm{m}^{2}$ (Flores et al., 2011).

En el Pacífico colombiano, las densidades presentaron valores variables. Para Tumaco entre 0.38 y 4.23 conchas $/ \mathrm{m}^{2}$ (Borda \& Cruz, 2004), Nariño de 0.3 a 1 conchas $/ \mathrm{m}^{2}$ (Espinosa,
Delgado Hernández, Orobio Riofrío, MejíaLadino, \& Gil-Agudelo, 2010) y en Málaga 0.5 a 0.8 conchas $/ \mathrm{m}^{2}$ (Lucero et al., 2012). Para Perú, se comunican valores variables entre 2.15 y 13 conchas $/ \mathrm{m}^{2}$ entre 1980 y 2015 , con una disminución de $48 \%$ de la densidad en 35 años (Ordinola, Alemán, Inga, Vera, \& Llanos, 2019). Para Ecuador, en Puerto el Morro, se comunican densidades de 4.3 conchas $/ \mathrm{m}^{2}$ en el 2006, 2.9 conchas $/ \mathrm{m}^{2}$ en el 2007 y 2.7 conchas $/ \mathrm{m}^{2}$ en el 2017 - 2018 (GamboaLandivar, 2019). Sin duda, la tendencia general en las poblaciones de concha negra, en Centro y Sur América, ha sido a una disminución en la densidad.

Al comparar las densidades estimadas para Panamá con el resto de las áreas de Centro y Suramérica, resulta que son de las más bajas de la región, sólo por encima de Playa Blanca en Costa Rica (Silva-Benavides \& Bonilla, 2015) y Bahía de Jiquilisco en el Salvador (Flores et al., 2011), contrario a lo que ocurre con las tallas, donde en Panamá se presentan los mayores valores de la región. Aunque no necesariamente los diseños de muestreo son comparables, lo seguro es que en toda la región existe una tendencia a la disminución de las densidades en los últimos años, acompañadas de una caída de las tallas en la mayoría de los países, excepto en el GM.

Las bajas densidades pueden estar asociada a diversos factores, entre ellos la presión pesquera, ya que los primeros ejemplares que desaparecen son los grandes, de tal suerte que la pesquería es sostenida por ejemplares pequeños. Para localidades en la zona del sur de Costa Rica la sobrepesca se manifiesta en la captura de tallas pequeñas, asociado sobre todo al pequeño tamaño de las poblaciones explotadas (Stern-Pirlot \& Wolff, 2006). Esta tendencia se manifiesta también en otras localidades de Centro y Sur América, donde se capturan ejemplares por debajo de la talla mínima legal, Manglar de Purruja en Costa Rica (Silva \& Bonilla, 2001), costa ecuatoriana (Mora \& Moreno, 2009), Bahía de Málaga, Pacífico colombiano (Lucero et al., 2012) y en Tumbes, Perú (Ordinola et al., 2019). También 
se refleja la sobrepesca en la caída de la captura por unidad de esfuerzo, es así como Lucero et al., (2012) comunican que ese indicador disminuyó alrededor de un $60 \%$ en 10 años para la Bahía de Málaga, Colombia. Otros factores responsables de estas bajas densidades son los procesos de contaminación del manglar, así como eventos climáticos regionales (El niño) y la destrucción del manglar (Borda \& Cruz, 2004; Lucero et al., 2012).

Un aspecto importante a considerar es que, a pesar de la caída de las densidades, la talla promedio de extracción, tanto para MD de David como para el GM se ha mantenido por encima de los $50 \mathrm{~mm}$ de LT, por encima de la talla mínima legal de captura establecida para Costa Rica (47 mm), Perú, El Salvador y Ecuador (45 mm), Nicaragua y Colombia $(50 \mathrm{~mm})$ (Silva \& Bonilla 2001; Borda \& Cruz, 2004; Mora \& Moreno, 2009; Espinosa et al., 2010), lo cual en término de sostenibilidad del recurso llevó a establecer como medida de manejo para el GM una talla mínima de extracción de $50 \mathrm{~mm}$ de longitud total, única localidad del Pacífico panameño con esta medida, producto de la aprobación del Plan de Manejo del área protegida.

El tener bajas densidades con tallas grandes se combina con indicadores poblacionales como la proporción sexual y el hermafroditismo que han variado de manera importante en los últimos años. La proporción sexual pasó de un macho por cada hembra en 1995 a casi cuatro hembras por cada macho y, de ausencia de hermafroditismo, a $3.9 \%$ de esta condición en el GM y $13.3 \%$ en MD (Robles y colaboradores, en preparación). En este sentido, los cambios en la proporción sexual y la presencia de hermafroditismo puede ser una respuesta poblacional a las bajas densidades del molusco, tanto en el GM como en MD.

Indicadores poblacionales como: estructura de tallas, proporción sexual, hermafroditismo y densidades, reflejan una respuesta de la población de concha negra a presiones, ya sea antropogénicas $\mathrm{y} / \mathrm{o}$ ambientales, sin embargo un factor para considerar son los eventos catastróficos que ocurren en el Golfo de Montijo y
Manglares de David, cada año, donde mueren gran cantidad de organismos marinos, vinculado principalmente a procesos de fumigación en sectores agrícolas que colindan con ambas áreas y que se reflejan sobre todo en la mortandad de peces (La Estrella de Panamá, 17 de junio de 2014, El Siglo, 9 de noviembre de 2018). Sin duda estos factores afectan con mayor intensidad los momentos más frágiles del ciclo reproductivo, las fases larvarias.

La distribución y abundancia de la concha negra en el manglar dependen de diferentes factores: estructura, composición y dureza del sustrato, especies arbóreas presentes, parámetros fisicoquímicos y eventos climáticos (Vega, 1994; Silva \& Bonilla, 2001; Guilbert, 2007; Silva-Benavides \& Bonilla, 2015; Ordinola et al., 2019). Para el Golfo de Montijo, donde se ha profundizado un poco más en estos aspectos, se determinó que se distribuye hasta salinidades tan bajas como 9 ups y tan altas como 39 ups, pero con mayor densidad entre 18 y 35 ups. En este sentido, Vega (1994) estableció en Térraba-Sierpe Costa Rica, que el piso de salinidad para la distribución de la concha negra es 12 ups, lo que coincide con resultados de ensayos que han demostrado que en el intervalo 15 a 33 ups la mortalidad de la concha negra fue nula durante el tiempo de experimentación, contrario a salinidades menores donde la mortalidad fue alta y la respuesta de los individuos incluían la disminución de la capacidad de filtración (Mendoza et al., 2017). Otros autores, han indicado que la salinidad es un factor importante en la distribución de la concha negra, y que localidades donde el aporte de agua dulce es abundante disminuye la densidad o no hay concha negra, a pesar de presentar sustratos adecuados para su desarrollo (Vega, 1994; Silva \& Bonilla, 2001, PantaVélez et al., 2020) y años con abundantes lluvias, por ejemplo durante eventos del Niño severos, provocaron mortalidad en la concha negra, contrario a localidades con salinidades altas donde la densidad del molusco fue alta (Ordinola et al., 2020).

Otros factores analizados son la densidad y las tallas de la concha negra en función de la 
distancia al borde del canal. En este sentido, se han comunicado diferentes resultados, entre los cuales están que la abundancia no mostró diferencias entre estratos (Ordinola et al., 2020), que la densidad aumenta hacia los estratos interiores de manglar (Vega, 1994; Mora-Sánchez, 2012) o que las densidades disminuyen en los estratos medios e internos (Guilbert, 2007; Flores et al., 2011), este último escenario coincide con nuestros resultados, donde se encontró que la densidad disminuye hacia las áreas cercanas a tierra firme.

De todos estos trabajos, los que analizan la distribución desde el borde del manglar con el canal principal hasta el límite del manglar con tierra firme son el presente estudio y el de Guilbert (2007), por lo que las diferencias entre los resultados de los diferentes estudios probablemente están asociado a un factor de escala, que se refleja en la utilización de diferentes diseños de muestreo, sobre todo las distancias consideradas entre estratos. Esto nos lleva definir que la concha negra, por lo menos en los manglares del GM, presentó un ámbito de distribución amplio, llegando hasta los límites del manglar con tierra firme, diferente a lo comunicado por Campos et al., (1990) y MacKenzie (2001) que mencionan que la concha negra se distribuye hasta los $200 \mathrm{~m}$ desde el borde del manglar hacia la parte interna del mismo.

La variación de las tallas en función de los estratos resultó según lo esperado, el aumento de estas hacia la parte interna del manglar, debido a que los extractores visitan estos sitios con menos frecuencia, lo que coincide con lo expuesto por Silva \& Bonilla (2001) para el Manglar de Purruja, Golfito, Costa Rica, al afirmar que los sitios con mayor visitación, presentaron menores tallas. Sin embargo, Ordinola et al., (2020) en Tumbes, Perú, no encuentran diferencias significativas entre estratos para esta variable y Flores et al., (2011), aunque no concluye al respecto, los promedios de tallas entre estratos para cada sitio de muestreo en Bahía de Jiquilisco, El Salvador, son muy similares, Puerto Ramírez 49.1, 49.0 y 49.0 mm; y el Jobal, 43.7, 43.8 y $43.7 \mathrm{~mm}$, en los estratos externo, medio e interno, respectivamente.
Para concluir, podemos afirmar que las tallas, densidades y distribución de la concha negra en el Golfo de Montijo y Manglares de David son el resultado, no solo de los procesos de explotación, sino que es consecuencia diferentes factores que inciden sobre estos parámetros. En este sentido es importante evaluar la contaminación ambiental, ya que puede estar incidiendo sobre estos parámetros.

Declaración de ética: los autores declaran que todos están de acuerdo con esta publicación y que han hecho aportes que justifican su autoría; que no hay conflicto de interés de ningún tipo; y que han cumplido con todos los requisitos y procedimientos éticos y legales pertinentes. Todas las fuentes de financiamiento se detallan plena y claramente en la sección de agradecimientos. El respectivo documento legal firmado se encuentra en los archivos de la revista.

\section{AGRADECIMIENTOS}

Se agradece el apoyo financiero de la Secretaria Nacional de Ciencia, Tecnología e Innovación de Panamá (SENACYT), a la Universidad de Panamá, a Conservación Internacional con el apoyo de la iniciativa Blue Action Fund y a la fundación Marviva por el apoyo dado a los proyectos que contribuyeron a generar la información para esta publicación. A los extractores de concha negra de Manglares de David y Golfo de Montijo por el apoyo en el levantamiento de datos. A los revisores que aportaron en la evaluación del documento.

\section{RESUMEN}

Introducción: Anadara tuberculosa es un recurso pesquero y económico importante, propio de los ecosistemas de manglar en el Pacífico Orienta Tropical. Objetivo: El objetivo del estudio fue analizar la estructura de tallas, distribución y densidad de $A$. tuberculosa, en el Golfo de Montijo y Manglares de David, para evaluar el estado del recurso en los dos sistemas de manglar del Pacífico de Panamá. Métodos: Los datos corresponden a información histórica de tallas y densidad levantada entre el 2004 y 2020 para el Golfo de Montijo (GM), así como datos de 
tallas, densidades y distribución levantados en el 2016 de manera simultánea en 51 cuadrantes en GM y 56 cuadrantes en Manglares de David (MD), muestreados en temporada seca, transición, lluviosa y transición, donde además se registró la temperatura, la salinidad y el pH del agua del canal y del agua retenida en el manglar. En el 2016 se instalaron y muestrearon cuadrantes fijos para seguimiento de densidades, muestreo que se repitió en el 2019. En el 2019 se establecen transectos desde el borde del manglar con el canal principal, hasta límite con tierra firme y en cada transecto se instalaron tres cuadrantes, en los estratos inicial, medio y final. Resultados: Los ejemplares del Golfo de Montijo resultaron más grandes, pero la densidad resultó superior en Manglares de David. La comparación de tallas por periodo de muestreo en el 2016, dentro de cada localidad, resultó significativa solo para el Golfo de Montijo, donde las mayores tallas se presentaron en marzo y junio y las menores en octubre-diciembre. No se encontró asociación entre la temperatura, salinidad y $\mathrm{pH}$, y la distribución del molusco. Para el Golfo de Montijo se encontró un descenso sostenido de las densidades desde 1.82 conchas/ $\mathrm{m}^{2}$ en el 2004 hasta 0.6 conchas $/ \mathrm{m}^{2}$ en el 2019 , contrario a lo observado en la estructura de tallas, donde se observó predominio de las tallas superiores a los $50 \mathrm{~mm}$ de longitud total y un aumento de la talla promedio de captura de $52.62 \mathrm{~mm}$ en el 2004 a $59.28 \mathrm{~mm}$ en el 2020 . Atendiendo a la distancia del canal principal, se determinó la presencia del molusco hasta poco más de dos kilómetros dentro del manglar y hasta más de $500 \mathrm{~m}$ del canal secundario más cercano, así como una disminución de la densidad y un aumento de la talla conforme nos acercamos a tierra firme. Conclusiones: Para A. tuberculosa, la combinación de una estructura de tallas sobre los $50 \mathrm{~mm}$ y de bajas densidades, indican que la población de concha negra en el Golfo de Montijo presenta un grado de deterioro, asociado a estresores pesqueros y ambientales.

Palabras clave: concha negra; Golfo de Chiriquí; pesquería; densidad; piangua.

\section{REFERENCIAS}

Bonilla, J.L., Robles, Y.A., \& Vega, A.J. (2013) Lengthweight relationship and biological information of the yellow snapper Lutjanus argentiventris from a tropical estuary: Río Caté, gulf of Montijo, Panama. Journal of Applied Ichthyology, 34, 227-229.

Borda, A.C., \& Cruz, R. (2004). Pesquería artesanal de bivalvos Anadara tuberculosa y Anadara similis y su relación con eventos ambientales. Pacífico colombiano. Revista de Investigaciones Marinas, 25(3),197-208.

Cámara, A.R., Díaz Olmo, F., Martínez, B., J.R., Morón, M. del C., Gómez-Ponce, C., Tabares, E., \& Vega, A.J. (2004). Directrices de gestión para la conservación y desarrollo integral de un humedal centroamericano: Golfo de Montijo (Litoral del Pacífico de Panamá). Ciudad de Panamá, Panamá: Autoridad Nacional del Ambiente-Agencia Española de Cooperación Internacional.

Campos, J.A., Fournier, M.L., \& Soto, R. (1990). Estimación de la población de Anadara tuberculosa (Bivalvia: Arcidae) en Sierpe Térraba, Costa Rica. Revista de Biología Tropical, 32(2b), 477-480.

CATHALAC (2007). Diagnóstico del estado actual de los manglares, su manejo y su relación con la pesquería en Panamá (Primera Etapa), Diagnósticos Biofísico, Institucional-Legal, Socioeconómico y Línea Base del Bosque de Manglar del Golfo de Montijo, Provincia de Veraguas, Informe Técnico Final, Centro del Agua del Trópico Húmedo para América Latina y el Caribe (CATHALAC). Ciudad de Panamá, Panamá: CATHALAC.

Cruz, R., \& Borda, C.A. (2003). Estado de explotación y pronóstico de la pesquería de la piangua Anadara tuberculosa (Sowerby, 1833) en el Pacífico colombiano. Revista de Investigaciones Marinas, 24, 221-230.

Cruz, R.A., \& Jiménez, J.A. (1994). Moluscos Asociados a la Áreas de Manglar de la Costa Pacífica de América Central. Heredia, Costa Rica: Editorial Fundación UNA.

Díaz del Olmo, F., Cámara-Artigas, R., \& Martínez-Batlle, J.R. (2004). Directrices y recomendaciones para el uso y gestión sostenible de los manglares de Chiriqui (República de Panamá). Ciudad de Panamá, Panamá: Autoridad Nacional del Ambiente-Agencia Española de Cooperación Internacional.

Espinosa, G., Delgado Hernández, M.F., Orobio Riofrío, B., Mejía-Ladino, L.M., \& Gil-Agudelo, D.L. (2010). Estado de la población y valoración de algunas estrategias de conservación del recurso piangua Anadara tuberculosa (Sowerby) en sectores de Bazán y Nerete, Costa Pacífica nariñense de Colombia. Boletín de Investigaciones Marinas y Costeras, 39(1), 161-176.

Flores, A.C., Romero, J.A., \& Córdova, M.F. (2011). Evaluación de la población de "curil" (Anadara tuberculosa) en los canales principales de puerto Ramírez y El Jobal, Bahía de Jiquilisco, Usulután, El Salvador (Tesis de Licenciatura). Universidad de El Salvador, El Salvador.

Gamboa-Landivar, L.M. (2019). Densidad y estructura poblacional de Anadara tuberculosa en Puerto el Morro: un análisis previo y posterior al establecimiento del área protegida (Tesis de Maestría). Universidad de Guayaquil, Ecuador.

Guilbert, A. (2007). State of the Anadara tuberculosa (Bivalvia: Arcidae) fishery in Las Perlas Archipielago, Panama (Tesis de Maestría). Heriot-Watt University, Reino Unido.

Jordán, L.Y., \& Gómez, J.A. (2006). Evaluación biológica de Anadara tuberculosa, Golfo de Montijo, República de Panamá. Tecnociencia, 8(2), 191-205. 
Keen, A.M. (1971). Sea shells of tropical West America ( $2^{\text {nd }}$ Ed.). California, Estados Unidos de América: Stanford University Press.

Lucero, C., Cantera, J., \& Neira, R. (2012). Pesquería y crecimiento de la piangua (Arcoida: Arcidae) Anadara tuberculosa en la Bahía de Málaga del Pacífico colombiano, 2005-2007. Revista de Biología Tropical, 60(1), 203-217.

Mackenzie, C. (2001). The Fisheries for Mangrove Cockles, Anadara spp., from Mexico to Peru, with Descriptions of their Habitats and Biology, the Fishermen's Lives, and the Effects of Shrimp Farming. Marine Fisheries Review, 63(1),1-39.

Mendoza, O., Pretell, K., Diringer, B., Avellan, R., Zapata, Z., Marchan, A., ... Mialhe, E. (2017). Respuesta fisiológica y molecular de Anadara tuberculosa (Arcoida: Arcidae) al estrés de salinidad. Revista de Biología Tropical, 65(3), 1142-1151.

Mora-Sánchez, E. (2012). Variación espacio temporal en la densidad y estructura de tallas y su relación con el esfuerzo pesquero de Anadara tuberculosa y Anadara similis (Pelecypoda: Arcidae) en el Archipiélago de Jambelí, Ecuador (Tesis de Maestría). Universidad de Guayaquil, Ecuador

Mora, E., \& Moreno, J. (2009). Recurso concha (Anadara tuberculosa y $A$. similis) en la costa ecuatoriana durante el 2004. Boletín Científico y Técnico, 20(1), $1-16$.

Ordinola, E., Alemán, S., \& Montero, P. (2020). Concha negra Anadara tuberculosa (Sowerby, 1833) y concha huequera Anadara similis (C.B. Adams, 1852) en los manglares de Tumbes. Setiembre 2018. Boletín Instituto del Mar del Perú, 47(1), 141-158.

Ordinola, E., Alemán, S., Inga, C.E., Vera, M., \& Llanos, J. (2019). Sinopsis biológica, poblacional y pesquera de Anadara tuberculosa (Sowerby, 1833) y Anadara similis (C.B. Adams, 1852) en los manglares de tumbes: 1995 a 2015. Boletín Instituto del Mar del Perú, 34(1), 223-264.

Panta-Vélez, R.P., Bermúdez-Medranda, A., Mero, P., Arrieche, D., \& Acosta-Balbás, V. (2020). Reproductive Cycle of Anadara tuberculosa (Sowerby, 1833) (Bivalvia: Arcidae) in a Mangrove System of the Chone River Estuary, Ecuador. Advances in Environmental Biology, 14(2), 1-11. DOI: 10.22587/ aeb.2020.14.2.1

Planeta. (2012). Ordenamiento de la extracción de la concha negra en los manglares de Alanje, David y San Lorenzo, Provincia de Chiriquí. Panamá, República de Panamá: Informe Inédito de la Fundación MarViva.

Silva, A., \& Bonilla, R. (2001). Abundancia y morfometría de Anadara tuberculosa y A. similis (Mollusca: Bivalvia) en el manglar de Purruja, Golfo Dulce,
Costa Rica. Revista de Biología Tropical, 49(2), 315-320.

Silva-Benavides, A., \& Bonilla, R. (2015). Estructura de la población y distribución de Anadara tuberculosa Sowerby (1833) (Mollusca: Bivalvia) en los manglares de Golfito y Playa Blanca de Puerto Jiménez, Golfo Dulce, Costa Rica. Revista de Biología Tropical, 63(1), 287-298.

Squires, H.J., Esteves, M., Barona, O., \& Mora, O. (1975). Mangrove cockles, Anadara spp. of the Pacific Coast of Colombia. Veliger, 18, 57-68.

Stern-Pirlot, A., \& Wolff, M. (2006). Population dynamics and fisheries potential of Anadara tuberculosa (Bivalvia: Arcidae) along the pacific coast of Costa Rica. Revista de Biología Tropical, 54, 87-99.

Tovar, D. (2008). Propuesta para el establecimiento de la nueva área protegida de los manglares del Golfo de Chiriqui (distritos de Alanje, David y San Lorenzo), Panamá. Ciudad de Panamá, Panamá: The Nature Conservancy.

Vega, A.J. (1994). Estructura de la población, rendimiento y épocas reproductivas de Anadara spp. (Bivalvia: Arcidae) en la Reserva Forestal Térraba-Sierpe, Puntarenas Costa Rica. Con recomendaciones para su manejo (Tesis de Maestría). Universidad de Costa Rica, Costa Rica.

Vega, A.J., Mena, C., \& Robles, P.Y. (2018). pesca artesanal de Cardisoma crassum (cangrejo) y Ucides occidentalis (mangote) en el sector nororiental del Golfo de Montijo, Pacífico de Panamá. Tecnociencia, 20(2), 5-22.

Vega, A.J., Quezada, F., \& Robles, Y.A. (2013). Aspectos biológicos y pesqueros de Scomberomorus sierra (Perciformes: Scombridae) en el Golfo de Montijo, Pacífico de Panamá. Tecnociencia, 15(2), 53-70.

Vega, A.J., Robles, Y., Boniche, S., \& Rodríguez, M. (2008). Aspectos biológicos-pesqueros del género Cynoscion (Pisces: Sciaenidae) en el Golfo de Montijo, Pacífico panameño. Tecnociencia, 10(2), 9-26.

Vega, A.J., Robles, Y.A., \& Gil, D. (2013). Biología y pesquería de Panulirus gracilis (Streeets 1871) (Decapoda: Palinuridae) en el Pacífico Occidental de Panamá. Revista Ciencias Marinas y Costeras, 5, 9-24

Vega, A.J., Robles, Y.A., \& Torres, L. (2014). Análisis biológico y pesquero de la extracción del poliqueto Americonuphis reesei en el Golfo de Montijo, Pacífico panameño. Tecnociencia, 16(2), 77-93.

Vega, A.J., Robles, Y.A., \& Godoy, K. (2015). El papel de los manglares como criaderos de pargo (Lutjanidae) en el Golfo de Chiriquí. Tecnociencia, 17(2), 111-125.

Zar, J.H. (2009). Biostatistical Analysis. New Jersey, Estados Unidos de América: Prentice Hall. 